\title{
Determining the Optimal Layout Design for Public Bicycle System within the Attractive Scope of a Metro Station
}

\author{
Jingxu Chen, ${ }^{1,2}$ Xuewu Chen, ${ }^{1}$ Hang Jiang, ${ }^{1}$ Senlai Zhu, ${ }^{1}$ Xiaowei Li, ${ }^{3}$ and Zhibin Li ${ }^{1}$ \\ ${ }^{1}$ Jiangsu Key Laboratory of Urban ITS, Southeast University, Si Pai Lou No. 2, Nanjing 210096, China \\ ${ }_{2}^{2}$ Jiangsu Province Collaborative Innovation Center of Modern Urban Traffic Technologies, Si Pai Lou No. 2, Nanjing 210096, China \\ ${ }^{3}$ School of Civil Engineering, Xian University of Architecture \& Technology, Yanta Road No. 13, Xi'an 710055, China \\ Correspondence should be addressed to Xuewu Chen; chenxuewu@seu.edu.cn
}

Received 11 July 2014; Accepted 7 September 2014

Academic Editor: Huimin Niu

Copyright (C) 2015 Jingxu Chen et al. This is an open access article distributed under the Creative Commons Attribution License, which permits unrestricted use, distribution, and reproduction in any medium, provided the original work is properly cited.

\begin{abstract}
Public bicycle acts as a seamless feeder mode in combination with the citywide public transit, as well as a competitor for the innercity short trips. The primary objective of this study is to address the layout planning of public bicycle system within the attracted scope of a metro station. Based on the land use function, population, and bicycle mode share, bicycle rental stations are divided into three types, namely, the metro station, district station, and resident station, and later the quantity of bicycle facilities in each rental station is estimated. Then, the service stations are selected from these bicycle rental stations to provide the service of periodical bicycle redistribution. An improved immune algorithm is proposed to determine the number and locations of service stations and the optimal route options for the implement of redistributing strategy. Finally, a case study of Nanjing Tianyin Road metro station is conducted to illustrate the proposed model and clarify some of its implementation details.
\end{abstract}

\section{Introduction}

Public bicycle system, also known as bicycle sharing system, has been introduced as both a competitor for one-way trips and a seamless feeder service to public transit. In the past decades, there has been a measureable increase in public bicycle use (shown in Figure 1) for both commuting and recreational trips in Europe [1-3], North America [4-8], and Asia $[9,10]$. At present there are an estimated 375 programs in approximately 165 cities worldwide with more than 237,000 bicycles in May 2011 [11]. Since public bicycle system was first launched in The Netherlands in 1965, its evolution has been categorized into four key generations [12]. The first generation is called white bicycle plan. Bicycles were unlocked and located haphazardly throughout the city. They could be picked up and off anywhere in the city, and their use was free of charge. The main disadvantage of first-generation system is the serious result of bicycle theft and vandalism. Second generation, the coin-deposit system, made some improvement based on the problems encountered in the first-generation program. The system was characterized by bicycles with locks and specific docking stations where bicycles were borrowed and returned. In a third generation of public bicycle system (the so-called ITbased system), advanced technology (e.g., magnetic-stripe cards, smartcards, or mobile phones) was introduced for check-in and check-out in docking stations. Information technology could improve system service and hinder bike theft. In this system, bicycles were in free use for a certain period of time (usually half an hour or an hour) and then were charged for the extra time to encourage short trips. The fourth generation is a demand-responsive, multimodal system, which is built on the technology of the thirdgeneration system by implementing enhanced features. The most innovative feature is bicycle redistribution which can facilitate system rebalancing. Based upon real-time demand, bicycles are moved from one station to another to ensure that bicycles and empty racks are always available for users pickup and drop-off at any station.

The history of public bicycle in China is relatively short. The largest and most famous public bicycle program in China is in Hangzhou. As of March 2011, Hangzhou public bicycle 


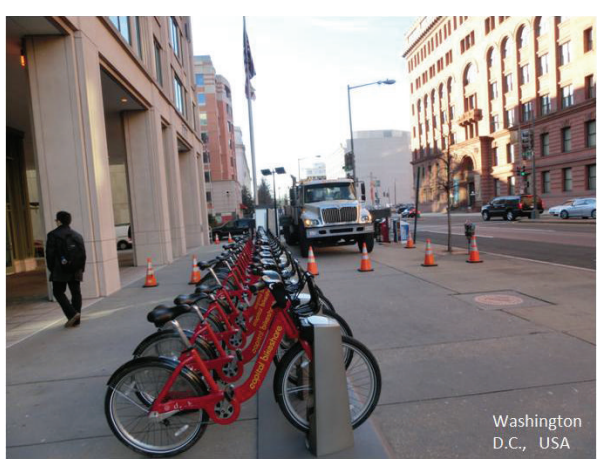

(a)

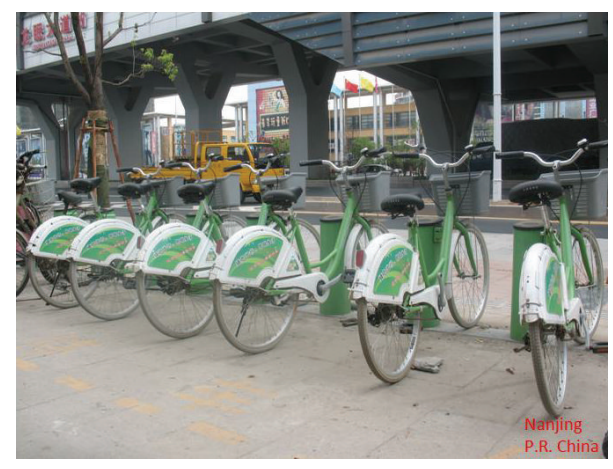

(b)

FIGURE 1: Typical public bicycle use and docking facility.

operated 60,600 bicycles with 2,416 stations [13]. Despite its limited experience, China is one of the fastest growing markets for public bicycle today. At present, more than sixty cities in China are developed or are developing public bicycle system [14]. Currently, public bicycle service provided in these cities is classified as the third-generation IT-based system, because it uses distinct bicycles with locks, docking stations, and smart cards for bicycle check-in and check-out. As a complement to existing public transit, the success of public bicycle system heavily depends on the locations of bicycle rental stations. In China, bicycle rental stations are usually constructed under the attractive scope of citywide transportation stations (e.g., metro, BRT, or light rail transit; the object of this study only refers to metro stations). Besides, in the vicinity of a metro station, there are usually many residential neighborhoods. They are the origins of current and potential public bicycle users. If bicycle rental stations are located in the entrance of each residential neighborhood, it is highly likely to generate an increase in the number of public bicycle users. However, few relative studies in the literature focus on the layout planning of public bicycle rental stations within the attracted scope of a specific public transit station.

In the future, the public bicycle system will evolve into the demand-responsive, multimodal system. At present, only some public bicycle systems are included in the category of the fourth generation, such as Montreal, Canada [15], and current research on the existing fourth-generation system is limited. For instance, an online survey was conducted in Montreal by Julie et al. [16] to determine the factors influencing frequency of public bicycle use. Results show that the proximity of home to docking stations had the greatest effect on the likelihood for use of a public bicycle system. As mentioned above, in the fourth-generation system, bicycles are redistributed among docking stations based on the realtime demand. Before implementing bicycle redistribution strategy, it is crucial to find proper number and locations of docking stations which can provide the service of dispatching bicycles. However, previous studies did not consider how to determine service stations in a public bicycle system. Besides, these previous studies still did not focus on the route options among various docking stations when redistributing public bicycles.
The primary objective of this study is to investigate the layout design of public bicycle rental stations within the attracted scope of a metro station. More specifically, this study includes the following two tasks: (1) to classify public bicycle rental stations around a metro station into three types and (2) to establish an improved immune algorithm to determine appropriate bicycle service stations and to search the optimal routes when redistributing public bicycles among various docking stations. The remainder of this paper is organized as follows. In Section 2, qualitative classification of public bicycle docking stations is presented and methodology used in this study to model the layout design of the public bicycle system is proposed. Section 3 gives a case study to illustrate the proposed model. The paper ends with brief concluding remarks in Section 4.

\section{Model Formulation}

2.1. Problem Description. The public bicycle system is designed within the attractive scope of a specific metro station (see Figure 2). Many residential neighborhoods are constructed around the metro station. These residential areas are the main origins of public bicycle users, and hence public bicycle rental stations are suitable to be placed at the entrance of each residential neighborhood. These docking stations are called resident stations, which are the main origin and destination of public bicycle in daily trips. Public bicycles from these resident stations are usually served as both an intermodal transfer to existing public transit and a competitor for the entire trip. Bicycle rental stations placed at the destination can be categorized into two types. (1) Metro station: the metro station is the most attractive location for public bicycle users to citywide area and hence is regarded as a central station. (2) District station: the flexibility of public bicycle makes it especially suitable for an entire short-distance trip. Residents can use public bicycles for commuting trips from home to their workplaces or for recreational trips to neighboring supermarkets, malls. Bicycle rental stations placed at these places are named district stations. The service stations are selected from these docking stations to provide the service of periodical bicycle redistribution. 


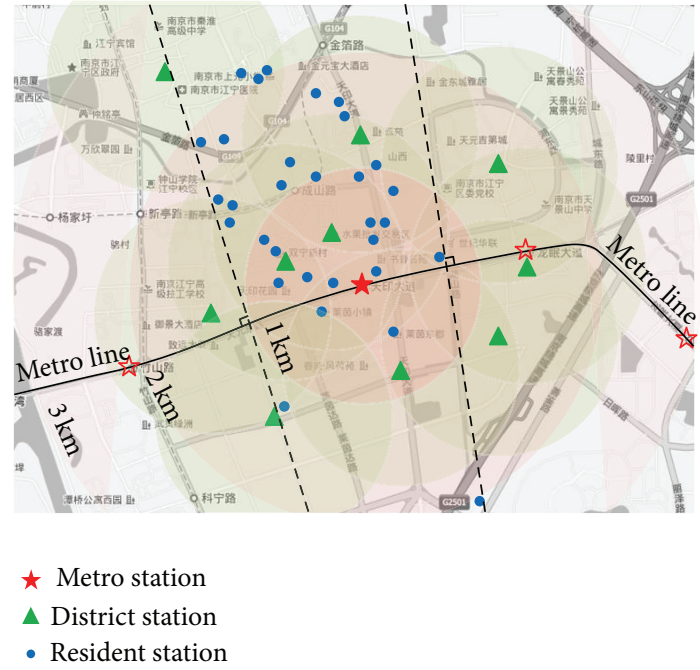

Figure 2: Public bicycle system within the attractive scope of a metro station.

The attractiveness of metro station (or district station) differs from the proximity from resident station to the metro station (or district station). Shan [17] proposed a bilevel structural model to get the relationship between bicycle mode share and trip distance. In two stages, the rational mode share of bicycle is represented in Table 1 .

Given a set of origins, destinations, the number of households in each residential neighborhood, and the stochastic travel demands from origin to destination with known parameters, we would like to determine bicycle facility placed at each docking station and where to locate the service stations. Specifically, resident stations should carry enough bicycles to guarantee residents can find public bicycles when needed, at least meeting the demand for morning peak period. The metro station and district stations provide corresponding bicycle racks for these public bicycles. Besides, bicycle rental stations should be checked up periodically. As the central station, the metro station mainly provides the service of dispatching bicycles for district stations and service stations. Each service station is responsible for some resident stations which belong to its management.

2.2. Mathematical Model. To formulate the problem, variables and parameters are first introduced as follows.

\section{Subscripts and Sets}

$i \in I$ denotes the resident stations;

$j \in J$ denotes the district stations;

$k \in K$ denotes the service stations.

\section{Input Parameters}

$Q$ is the daily bicycle demand within the morning peak period;

$Q_{A}$ is the number of public bicycles in the metro station;
$Q_{B}$ is the number of public bicycles in the district stations;

$Q_{C}$ is the number of public bicycles in the resident stations;

$\omega_{i A}$ is the bicycle mode share from station $i$ to the metro station;

$\omega_{i j}$ is the bicycle mode share from station $i$ to station $j$;

$p_{i j}$ is the ratio of bicycles (from station $i$ to station $j$ ) to total bicycles in station $i$;

$k_{g}$ is the ratio of public bicycles to total bicycles;

$k_{\omega_{i}}$ is the bicycle mode share in station $i$;

$d_{i k}$ is the distance from station $i$ to station $k$;

$d_{i A}$ is the distance from station $i$ to the metro station;

$s$ is the upper limit distance between two stations;

$n$ is the number of service stations;

$\delta_{i k}$ equals 1 if station $i$ is dominated by service station $k$ and is 0 otherwise;

$\sigma_{i}$ equals 1 if station $i$ is a service station and is 0 otherwise.

\section{Algorithm Variables}

$u, v \in N$ denotes the antibodies;

$\mathrm{N}$ is the capacity of a memory base;

$P_{u}$ is the expected antibody-production probability of antibody $u$;

$F_{u}$ is the affinity between antigen and antibody $u$;

$F_{u v}$ is the affinity between antibody $u$ and antibody $v$;

$C_{u}$ is the antibody concentration;

$C_{p}$ is the penalty coefficient when distance exceeds $s$;

$\alpha$ is a preset constant between 0 and 1 ;

$\beta$ is a preset threshold value.

The number of public bicycles placed at each resident station should at least meet the peak-hour travel demand for each residential neighborhood. Accordingly, the metro station and district stations should have enough empty bicycle racks exclusively available for these public bicycles. In other words, supply of public bicycle facility in the metro station and district stations equals public bicycles in resident stations, which is expressed as follows:

$$
Q_{A}+\sum_{j \in J} Q_{B_{j}}=\sum_{i \in I} Q_{C_{i}}
$$


TABle 1: Bicycle mode share for different trip distance.

\begin{tabular}{lcccccccc}
\hline Trip distance $(\mathrm{km})$ & {$[0,1)$} & {$[1,2)$} & {$[2,3)$} & {$[3,4)$} & {$[4,5)$} & {$[5,6)$} & {$[6,7)$} & {$[7,+\infty)$} \\
\hline Two stages & & & & & & & & \\
$\quad$ User equilibrium (\%) & 19.97 & 56.77 & 72.78 & 54.18 & 42.33 & 18.8 & 12.28 \\
System optimum (\%) & 19.97 & 53.32 & 62.5 & 33.45 & 19.18 & 3.82 & 2.3 & 0 \\
\hline
\end{tabular}

The number of public bicycles in the resident stations and the number of bicycles racks in the metro station and district stations are described by the following:

$$
\begin{aligned}
Q_{A} & =\sum_{i \in I} \frac{\omega_{i A} Q_{C_{i}}}{\omega_{i A}+\sum_{j \in J} p_{i j} \omega_{i j}}, \\
Q_{B_{j}} & =\sum_{i \in I} \frac{p_{i j} \omega_{i j} Q_{C_{i}}}{\omega_{i A}+\sum_{j \in J} p_{i j} \omega_{i j}}, \\
Q_{C_{i}} & =k_{g} \cdot k_{\omega_{i}} \cdot Q_{i}, \\
k_{\omega_{i}} & =\frac{\omega_{i A}+\sum_{j \in J} p_{i j} \omega_{i j}}{1+J} .
\end{aligned}
$$

When the public bicycle system is established, we need service stations to manage docking stations periodically, such as inspecting the problems about theft or vandalism and dispatching bicycles to redistribute bicycles among docking stations. As the central station, the metro station will be a service station responsible for managing the metro station and district stations. Within resident stations, several docking stations are selected to provide the service of management for certain resident stations. Based on the abovementioned notation, the following service station model can be formulated:

$$
\min F=\sum_{i \in I} \sum_{k \in K} Q_{C_{i}} d_{i k} \delta_{i k}+\sum_{i \in I} Q_{A} d_{i A} \sigma_{i},
$$

subject to

$$
\begin{gathered}
d_{i k} \leq s \quad \forall i \in I, \quad \forall k \in K, \\
\sum_{k \in K} \delta_{i k}=1 \quad \forall i \in I, \\
\delta_{i k} \leq \sigma_{i}, \quad \forall i \in I, \quad \forall k \in K, \\
\delta_{i k} \in\{0,1\}, \quad \forall i \in I, \quad \forall k \in K, \\
\sigma_{i} \in\{0,1\}, \quad \forall i \in I .
\end{gathered}
$$

The objective function (3) contains two terms. The first term $F_{1}$ is the sum of the product of travel demand of resident station and proximity from resident station to the service station. The second term $F_{2}$ is the sum of the product of travel demand of metro station and proximity from metro station to the service stations. Constraint (4) ensures that the proximity from the resident station to the service station is no more than the upper limit distance $s$. Constraints (5) and (6) ensure that each resident station is only dominated by one service station. Constraints (7) and (8) are the integrality requirements for the location variables of the service stations.

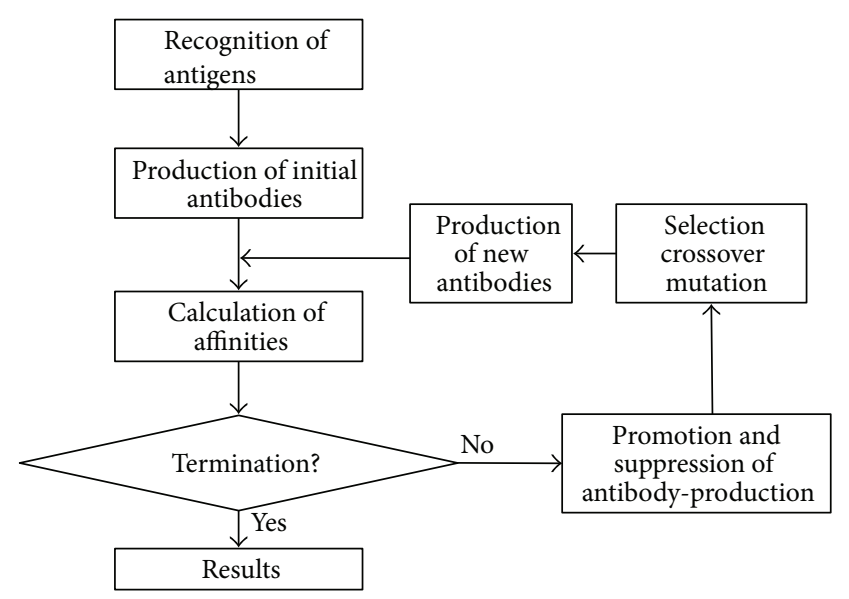

FIgURE 3: Flow chart of immune algorithm.

The service station model is an integer nonlinear program. In this paper, an improved immune algorithm (IIA) is applied to determine the optimal number and locations of the service stations within the resident stations. IIA is composed of six steps shown in Figure 3.

The process of IIA is described below.

Step 1 (recognition of antigens). Input data, the objective function (3) and constraints (4)-(8), operate as the antigens of immune algorithm.

Step 2 (production of initial antibodies). Establish a memory base, which contains $N$ antibodies. Each antibody consists of $n$ service stations. Initially, antibodies are created randomly from the resident stations.

Step 3 (calculation of affinities). Each antibody is estimated by the expected antibody-production probability $P . P$ is related with the affinity between antigen and antibody $F_{u}$ and antibody concentration $C_{u}$. Calculation of three variables is expressed as follows:

$$
\begin{gathered}
F_{u}=1 \times\left(\sum_{i \in I} \sum_{k \in K} Q_{C_{i}} d_{i k} \delta_{i k}+\sum_{i \in I} Q_{A} d_{i A} \sigma_{i}\right. \\
\left.+C_{P} \sum_{i \in I} \min \left(1-\sum_{k \in K} \delta_{i k}, 0\right)\right)^{-1}, \\
C_{u}=\frac{1}{N} \sum_{v \in N} F_{u v}, \\
P_{u}=\alpha \frac{F_{u}}{\sum_{v \in N} F_{v}}+(1-\alpha) \frac{C_{u}}{\sum_{v \in N} C_{v}},
\end{gathered}
$$


where $F_{u v}$ is calculated based on the method of R-contiguous bits matching [18]. $F_{u v}$ equals 1 if $F_{u v}$ is larger than $\beta$ and is 0 otherwise.

Step 4 (sequence of antibody-production). For advance of the optimal performance, $N$ antibodies are descending ordered based on their values of $P$.

Step 5 (production of new antibodies). In this step, new antibodies will be produced by the operations of selection, crossover, and mutation. Antibodies are selected into the memory base, based on roulette wheel selection. Two antibodies selected randomly are mutated by the predetermined mutation rate. And the linear crossover is carried on each other. These new antibodies will substitute initial antibodies with low $P$.

Step 6 (termination criterion). If the termination criterion is satisfied, this optimization procedure ends. The termination criterion is the number of generations which attains the predetermined iteration number, which is assumed to be 200 . If it is not satisfied, repeat from Step 3 to Step 5 until the terminal condition is met.

\section{Case Study Analysis}

3.1. Data Collection and Settings. A small example was used to illustrate the proposed model. In this paper, Tianyin Road station, a stereotypical metro station from Nanjing metro line 1 , was selected as the central metro station. It is located in Jiangning District, which is an important district of Nanjing city and owns the largest population of all the districts. Tianyin Road station has large daily travel demand for the downtown district. Besides, many supporting facilities for employment, living, and amusement are surrounded by this area. 10 sites are selected as district stations, and these sites are distributed from each direction around the metro station. There are 31 residential neighborhoods within the radiation scope of Tianyin Road station, and 31 district stations are set, respectively. These district stations are located at the entrance of each residential neighborhood. Locations of the bicycle rental stations are shown in Figure 2.

Data about number of households in each residential area were obtained. Initial planning of public bicycle system should be conservative, and we assume the average number of travel trips for each household is 1 . The percentage of public bicycles in total bicycles is assumed to be 0.2 . The parameters in the improved immune algorithm (IIA) are set as follows: IIA is run with a population size of 50 and the number of evolution generations is set to 200 . Crossover probability and mutation probability are set to be 0.7 and 0.3 . $\alpha$ and $\beta$ are set to be 0.9 and 0.7 , respectively. As shown in Table 1, the bicycle mode share decreases when distance is over $3 \mathrm{~km}$, and $s$ is set to be $3 \mathrm{~km}$. $C_{p}$ equals threefold $d_{i k}$.

3.2. Public Bicycle Facilities in Bicycle Rental Stations. The problem was solved by Matlab R2008b, on a notebook computer (Intel Core Duo and 1GB of memory) with a Microsoft Windows XP operating system. Table 2 presents

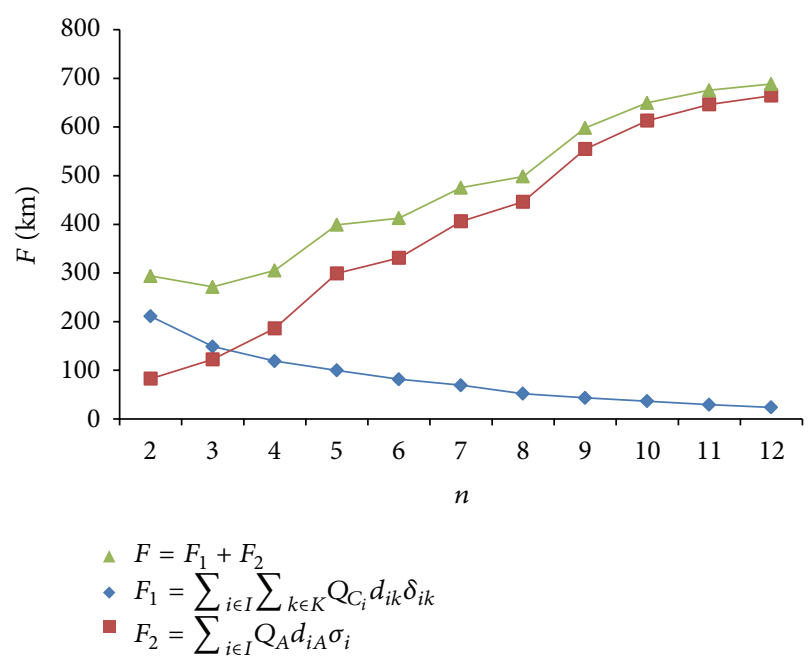

FIGURE 4: Relation between number of service stations and objective function value $F$.

the number of public bicycles and corresponding racks in three types of bicycle rental stations. Public bicycles in resident stations will be allocated about 17 public bicycles per thousand households. During the initial planning stage, some resident stations (e.g., $\mathrm{C}_{3}, \mathrm{C}_{7}$, and $\mathrm{C}_{20}$ ) only set two or three bicycles, which will be the initiation of advocating residents understanding and utilizing public bicycles. When residents get familiar with the public bicycle system, it will be considered to gradually increase the number of public bicycles in these resident stations.

3.3. Results of Service Stations Selection. When bicycle facilities in each bicycle rental station are determined, service stations are needed to manage docking stations regularly, such as inspecting the problems about theft or vandalism and dispatching bicycles to rebalance bicycles among docking stations. As the central station, the metro station will be a service station responsible for managing the district station and service stations. Besides, IIA is utilized to determine the optimal number and locations of the service stations from the resident stations.

Figure 4 presents the relationship between the number of service stations and two terms in function (3). The sum of the product of proximity and demand in resident stations $F_{1}$ tends to be downward when the number of service stations increases, whereas the sum of the product of proximity and demand between the metro station and resident stations $F_{2}$ rises. When the number of service stations is 3 , the value of the objective function (3) reaches minimum. It means that the most suitable number of service stations is three within the attractive scope of Tianyin Road metro station.

The metro station mainly provides the service of dispatching bicycles for district stations and service stations. Each service station is responsible for some resident stations which belong to its management, as shown in Figure 5. 
TABLE 2: Bicycle facilities in bicycle rental stations.

\begin{tabular}{|c|c|c|c|c|c|c|c|c|c|}
\hline \multirow{2}{*}{ Type } & \multirow{2}{*}{ Sum } & \multicolumn{8}{|c|}{ The number of public bicycle facilities in each station } \\
\hline & & Station & Number & Station & Number & Station & Number & Station & Number \\
\hline Metro station & 147 & A & 147 & & & & & & \\
\hline \multirow{3}{*}{ District station } & \multirow{3}{*}{217} & $\mathrm{~B}_{1}$ & 12 & $\mathrm{~B}_{2}$ & 24 & $\mathrm{~B}_{3}$ & 19 & $\mathrm{~B}_{4}$ & 28 \\
\hline & & $\mathrm{B}_{5}$ & 27 & $\mathrm{~B}_{6}$ & 25 & $\mathrm{~B}_{7}$ & 20 & $\mathrm{~B}_{8}$ & 17 \\
\hline & & $\mathrm{B}_{9}$ & 24 & $\mathrm{~B}_{10}$ & 21 & & & & \\
\hline \multirow{8}{*}{ Resident station } & \multirow{8}{*}{364} & $\mathrm{C}_{1}$ & 13 & $\mathrm{C}_{2}$ & 21 & $\mathrm{C}_{3}$ & 3 & $\mathrm{C}_{4}$ & 6 \\
\hline & & $\mathrm{C}_{5}$ & 11 & $\mathrm{C}_{6}$ & 19 & $\mathrm{C}_{7}$ & 2 & $\mathrm{C}_{8}$ & 36 \\
\hline & & $\mathrm{C}_{9}$ & 38 & $\mathrm{C}_{10}$ & 7 & $\mathrm{C}_{11}$ & 5 & $\mathrm{C}_{12}$ & 4 \\
\hline & & $\mathrm{C}_{13}$ & 6 & $\mathrm{C}_{14}$ & 25 & $\mathrm{C}_{15}$ & 11 & $\mathrm{C}_{16}$ & 6 \\
\hline & & $\mathrm{C}_{17}$ & 5 & $\mathrm{C}_{18}$ & 10 & $\mathrm{C}_{19}$ & 14 & $\mathrm{C}_{20}$ & 3 \\
\hline & & $\mathrm{C}_{21}$ & 30 & $\mathrm{C}_{22}$ & 18 & $\mathrm{C}_{23}$ & 6 & $\mathrm{C}_{24}$ & 4 \\
\hline & & $\mathrm{C}_{25}$ & 14 & $\mathrm{C}_{26}$ & 10 & $\mathrm{C}_{27}$ & 6 & $\mathrm{C}_{28}$ & 6 \\
\hline & & $\mathrm{C}_{29}$ & 5 & $\mathrm{C}_{30}$ & 13 & $\mathrm{C}_{31}$ & 7 & & \\
\hline
\end{tabular}

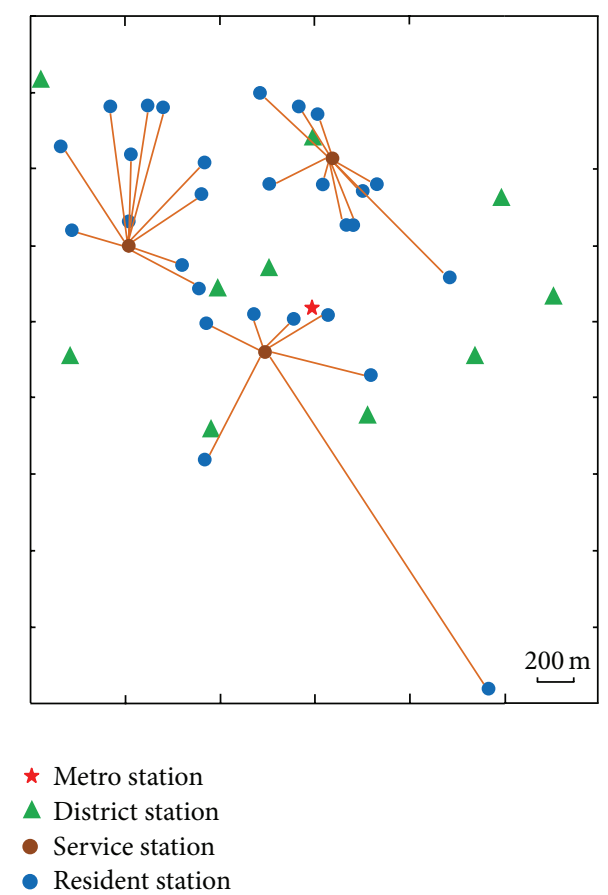

FIGURE 5: Public bicycle service stations and corresponding managed docking stations.

3.4. Optimal Routes for Regular Redistribution. Once the service stations are determined and the public bicycle system is put into use, bicycles need to be regularly redistributed from one station to another to ensure that bicycles and empty racks are always available for residents pick-up and drop-off at any station. The metro station mainly provides the service of dispatching bicycles for district stations and service stations. Each service station is responsible for certain resident stations which belong to its management. Thus, it is crucial to explore the optimal route options for implementing periodical redistribution, which is a traveling salesman problem (TSP). TSP is a NP-complete problem and it needs to be solved by some intelligent algorithms [19-21]. This paper utilized the discrete particle swarm algorithm [22] to search the optimal routes.

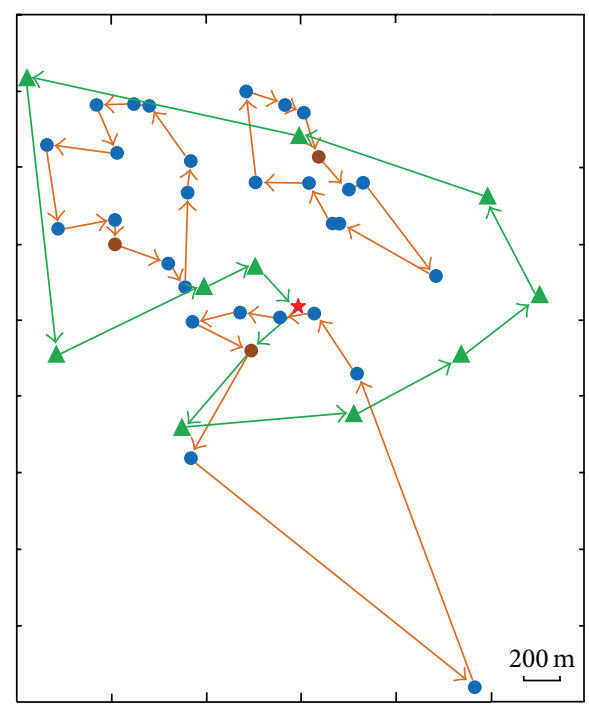

\footnotetext{
$\star$ Metro station

District station

- Service station

- Resident station
}

FIGURE 6: Optimal routes for the implement of redistributing strategy.

Figure 6 illustrates the optimal fixed-routes for redistributing services in Tianyin Road metro station. Three yellow routes, respectively, present the optimal route option for each service station and their managed resident stations. The green route covers all district stations and service stations and the redistributing strategy starts at the metro station and finally returns to this central station.

\section{Conclusions and Recommendations}

In recent years, public bicycles have received a lot of attention. Public bicycles serve as both a competitor for the innercity short-distance trips and a complementary feeder mode with citywide transportation to solve the "last mile" problem. 
This study investigates the layout design of public bicycle rental stations within the attracted scope of a metro station. Public bicycle rental stations are qualitatively classified into three types, namely, the metro station, district stations, and resident stations. Initial planning of the number of public bicycles placed at each resident station should meet the peak-hour travel demand for each residential neighborhood. Correspondingly, the metro station and district stations provide enough bicycle racks for these public bicycles docking. Besides, public bicycles in these bicycle rental stations should be redistributed periodically. The metro station redistributes bicycles among district stations and service stations. Meanwhile, service stations provide the dispatching bicycle service for certain resident stations which belong to their management. This paper has developed a mathematical model that provides such an integrated view. A case study of a typical metro station in Nanjing, China, is performed to illustrate the proposed model. The contributions of this paper to the literature are as follows: (1) most of current studies on public bicycle system in the literature have focused on better understanding and promotion policy issues, and we have not found any study that has addressed the strategic planning of the public bicycle rental stations based upon the attractive scope of a metro station; (2) this paper first proposes the service stations and establishes a model to address the selection of service stations and optimal routes for redistributing public bicycles. These findings will make a preparation for the implement of the fourth demandresponsive generation.

Future research would be useful in at least the three following directions. First, more data could be collected from various functional metro stations (e.g., dwelling, shopping, and industry) in other cities of countries. The similar procedure can be followed to obtain different layout designs of the public bicycle systems within the attractive scope of these metro stations. Specific public bicycle facility design would be implemented once the public bicycle system is established. Second, the reuse of public bicycles is not considered, which makes the calculation of the number of public bicycles at rental stations conservative. It would be helpful to develop a more accurate estimation through taking account of reuse rate. Third, the travel demands may vary each day. Thus, it would be helpful to develop a dynamic model to implement redistributing strategy based on the real-time demand (e.g., update the optimal route options daily). The authors recommend that future studies could focus on these issues.

\section{Disclosure}

This work was submitted for Presentation at the 5th International Conference on Green Intelligent Transportation System and Safety.

\section{Conflict of Interests}

The authors declare that there is no conflict of interests regarding the publication of this paper.

\section{Acknowledgments}

This research is supported by the Key Project of National Natural Science Foundation of China (no. 51338003), the National Key Basic Research Program (NKBRP) of China (no. 2012CB725400), and the National Natural Science Foundation of China (no. 51178109). The authors thank those students from the Southeast University who participated in data processing.

\section{References}

[1] S. A. Shaheen, A. P. Cohen, and E. W. Martin, "Public bikesharing in North America: early operator understanding and emerging trends," in Proceedings of the 92th Annual Meeting of the Transportation Research Board, Washington, DC, USA, February 2013.

[2] N. Lathia, S. Ahmed, and L. Capra, "Measuring the impact of opening the London shared bicycle scheme to casual users," Transportation Research Part C: Emerging Technologies, vol. 22, pp. 88-102, 2012.

[3] S. D. Parkes, G. Marsden, S. A. Shaheen, and A. P. Cohen, "Understanding the diffusion of public bikesharing systems: evidence from Europe and North America," Journal of Transport Geography, vol. 31, pp. 94-103, 2013.

[4] L. K. Maurer, "Feasibility study for a bicycle sharing program in Sacramento, California," in Proceedings of the 91st Annual Meeting of the Transportation Research Board, Washington, DC, USA, 2012.

[5] S. A. Shaheen, E. Martin, A. Cohen, and R. Finson, "Public bikesharing in North America: early operator and user understanding," MTI Report 11-26, MTI, San Jose, Calif, USA, 2012.

[6] G. R. Krykewycz, C. M. Puchalsky, J. Rocks, B. Bonnette, and F. Jaskiewicz, "Defining a primary market and estimating demand for major bicycle-sharing program in Philadelphia, Pennsylvania," Transportation Research Record: Journal of the Transportation Research Board, vol. 1, no. 2134, pp. 117-124, 2010.

[7] J. Gregerson, J. Hepp-Buchanan, D. Rowe et al., Seattle Bicycle Share Feasibility Study, Department of Urban Design and Planning, University of Washington College of Built Environment, 2011.

[8] A. Rixey, "Station-level forecasting of bike sharing ridership: station network effects in three US systems," in Proceedings of the 92th Annual Meeting of the Transportation Research Board, Washington, DC, USA, 2013.

[9] J.-R. Lin and T.-H. Yang Ta-Hui, "Strategic design of public bicycle sharing systems with service level constraints," Transportation Research Part E: Logistics and Transportation Review, vol. 47, no. 2, pp. 284-294, 2011.

[10] S. A. Shaheen, S. H. Guzaman, and H. Zhang, "Bikesharing in Europe, the Americas, and Asia past, present, and future," Transportation Research Record: Journal of the Transportation Research Board, vol. 1, no. 2143, pp. 159-167, 2010.

[11] R. Gleason and L. Miskimins, Exploring Bicycle Options for Federal Lands: Bike Sharing, Rentals and Employee Fleets, Federal Highway Administration, Washington, DC, USA, 2012.

[12] P. DeMaio, "Bike-sharing: history, impacts, models of provision, and future," Journal of Public Transportation, vol. 12, no. 4, pp. 41-56, 2009.

[13] S. A. Shaheen, H. Zhang, E. Martin, and S. Guzman, "China’s Hangzhou public bicycle: understanding early adoption and 
behavioral response to bikesharing," Transportation Research Record, no. 2247, pp. 33-41, 2011.

[14] Y. Zhang, X. Liu, and H. Zhang, "Urban public bicycle system in China: current situation, issues and countermeasures," China Development, vol. 13, pp. 74-80, 2013 (Chinese).

[15] C. Morency, M. Trépanier, and F. Godefroy, "Insight into Montreal's Bikesharing system," in Proceedings of the 90th Annual Meeting of the Transportation Research Board, Washington, DC, USA, 2011.

[16] B. Julie, H. Brian, and M. Ahmed, "Better understanding of factors influencing likelihood of using shared bicycle systems and frequency of use," Transportation Research Record: Journal of the Transportation Research Board, no. 2314, pp. 66-71, 2012.

[17] X. Shan, A research on urban bicycle transportation rational ridership and road resource allocation [Ph.D. thesis], Southeast University, Nanjing, China, 2007.

[18] X. Y. Liu, A. L. Zhang, Y. L. Gao, and W. Zhao, "A novel hybrid immune algorithm and its convergence based on the steepest descent algorithm," Applied Mathematics and Computation, vol. 218, no. 4, pp. 1291-1296, 2011.

[19] H.-Y. Yun, S.-J. Jeong, and K.-S. Kim, "Advanced harmony search with ant colony optimization for solving the traveling salesman problem," Journal of Applied Mathematics, vol. 2013, Article ID 123738, 8 pages, 2013.

[20] C. Moon, J. Kim, G. Choi, and Y. Seo, "An efficient genetic algorithm for the traveling salesman problem with precedence constraints," European Journal of Operational Research, vol. 140, no. 3, pp. 606-617, 2002.

[21] J. Sung and B. Jeong, "An adaptive evolutionary algorithm for traveling salesman problem with precedence constraints," The Scientific World Journal, vol. 2014, Article ID 313767, 11 pages, 2014.

[22] G. He and N. Huang, "A modified particle swarm optimization algorithm with applications," Applied Mathematics and Computation, vol. 219, no. 3, pp. 1053-1060, 2012. 


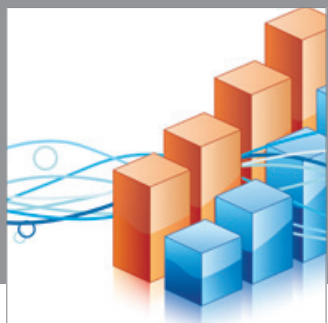

Advances in

Operations Research

mansans

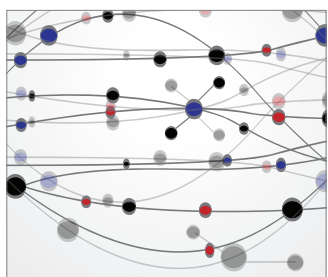

The Scientific World Journal
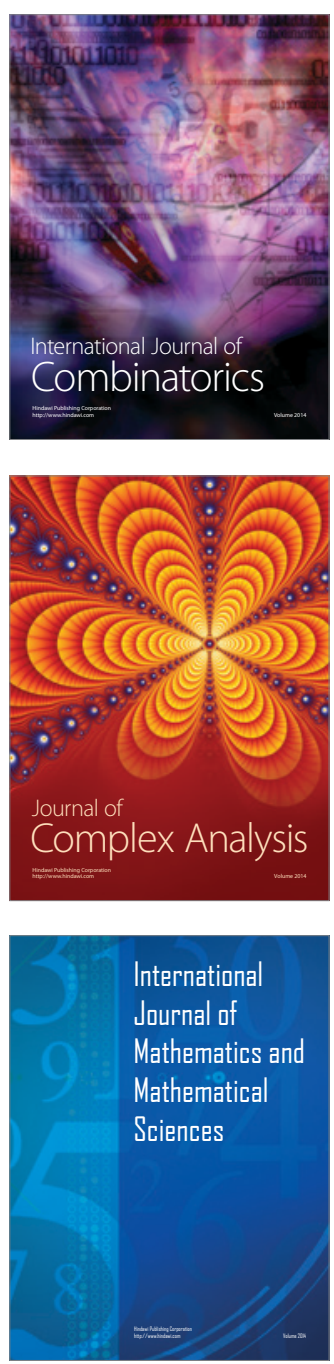
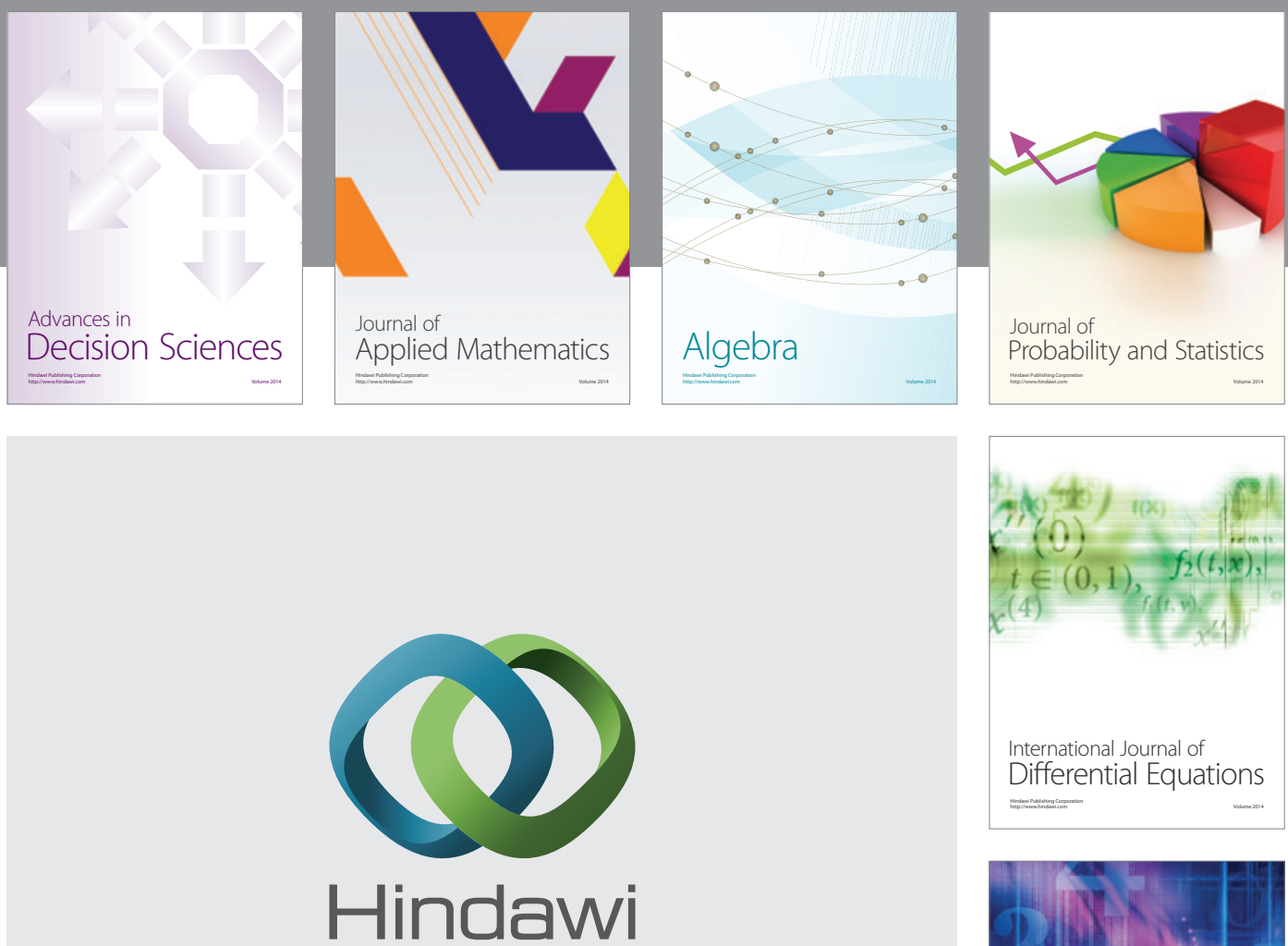

Submit your manuscripts at http://www.hindawi.com
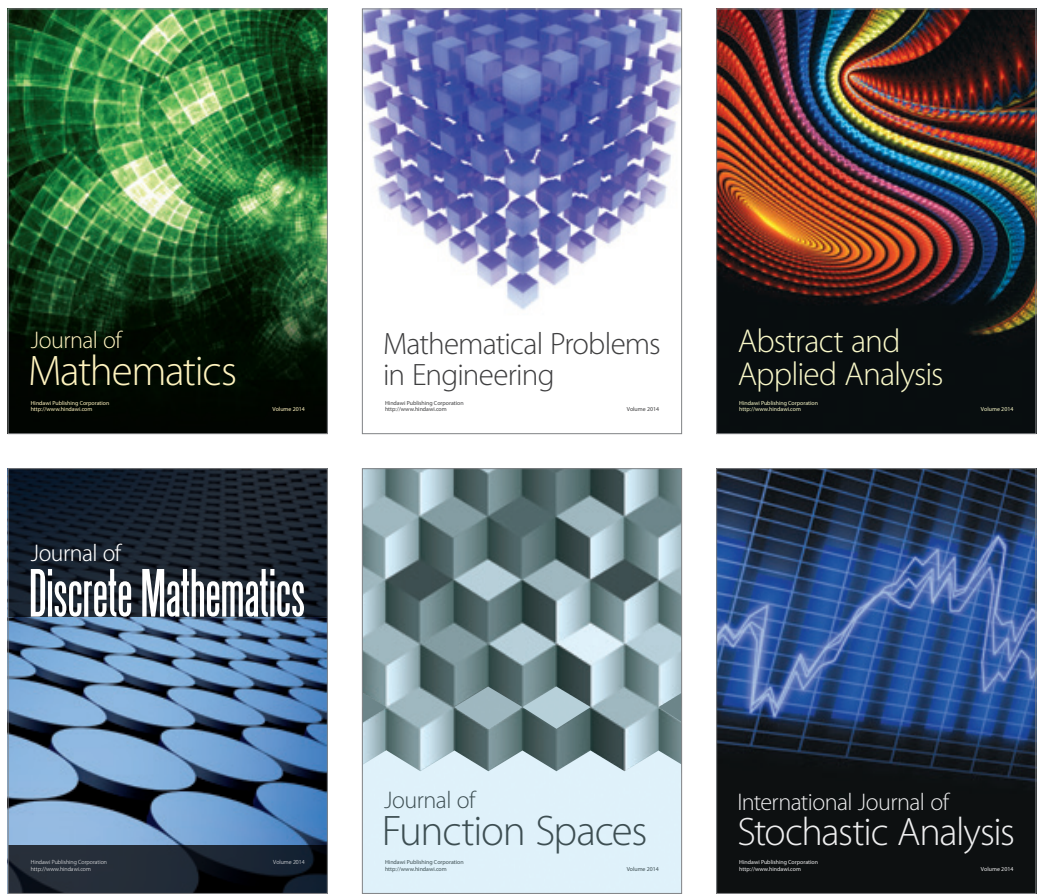

Journal of

Function Spaces

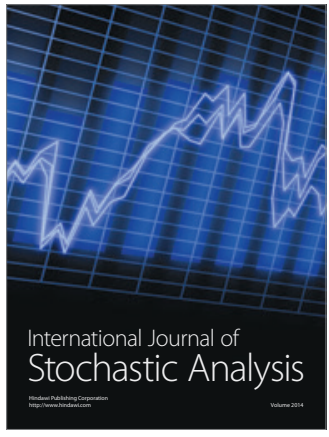

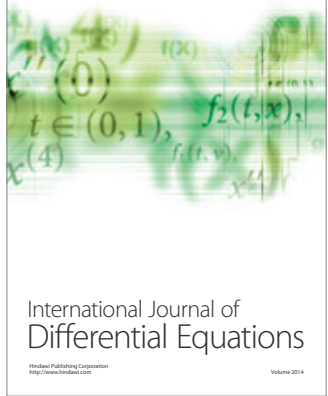
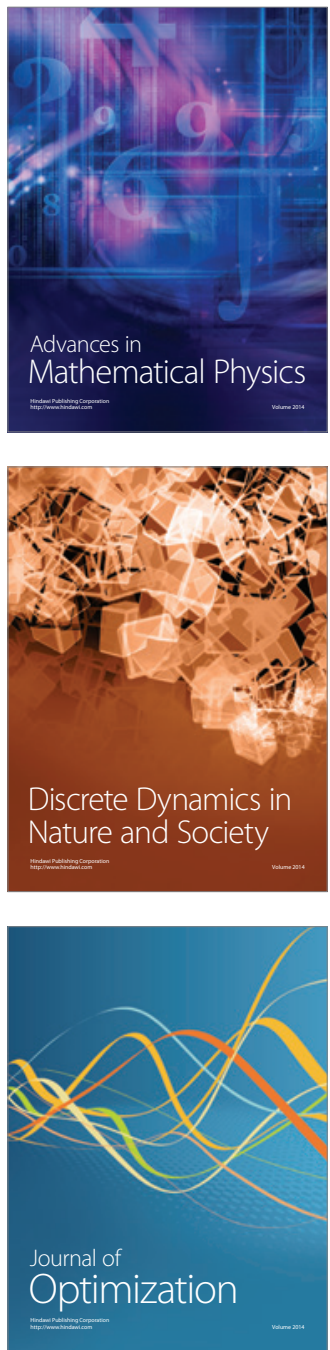